\title{
Progress in Blind Separation of Magnetoencephalographic Data
}

\author{
Barak A. Pearlmutter* Santiago Jaramillo*
}

\begin{abstract}
The match between the physics of MEG and the assumptions of the most well developed blind source separation (BSS) algorithms (unknown instantaneous linear mixing process, many sensors compared to expected recoverable sources, large data limit) have tempted researchers to apply these algorithms to MEG data. We review some of these efforts, with particular emphasis on our own work.
\end{abstract}

\section{Introduction}

Magnetoencephalographic (MEG) functional brain imaging is passive, and under ideal conditions can monitor the activation of a brain region with $\mathrm{mm}$ spatial resolution at a very high temporal resolution (Hämäläinen et al., 1993; George et al., 1995). The advantage of MEG over EEG comes mainly from the fact that the head is practically transparent to magnetic fields, but alters current flow substantially (Hari, 1999; Hari et al., 1997).

Typical signals associated with neuronal activity are on the order of one hundred $\mathrm{fT}$, while the noise signals within a shielded room tend to be much larger (Lewine and Orrison, 1995). Furthermore, the intrinsic sensor noise is comparable in magnitude to small neuronal signals. Therefore, what the sensors record during an experiment is always a mixture of small neuromagnetic and large noise signals.

The general goal of localizing and recovering the time course of these neuronal sources thus requires separating the signals from noise. The traditional approach consists of: (1) designing experiments that activate only a few regions, (2) averaging over trials, and (3) analysis making strong use of a mathematical model of propagation through the head. Having this model, a gradient method is applied to find fixed locations and time courses of a handful of dipoles to match (in least squares sense) the data. The problem with this technique is that the forward model is both ill conditioned and inexact, so the inverse estimate can have an unacceptable error. Furthermore, the validity of the results are hard to verify because they are forced by this procedure to be consistent with the understood physics of the situation, i.e. the forward model.

An alternative solution is to use blind source separation (BSS) techniques, as shown schematically in Figure 1 . The problem addressed by blind source separation

\footnotetext{
${ }^{*}$ Hamilton Institute, NUI Maynooth, Co. Kildare, Ireland.
}

(or equivalently for our purposes Independent Component Analysis, ICA), is that of segregating unobservable or latent source signals using only the information from the observed mixed signals. The BSS algorithms of interest here assume that the mixing process is linear, instantaneous, and unchanging. Such methods have been successfully applied to EEG (Jung et al., 1999, 2000) and FMRI (McKeown et al., 1998a,b). Due to its success in these domains, a number of groups began applying BSS to MEG.

Vigário et al. (1998, 2000) presented tantalizing results by applying BSS to MEG data. The main issue in their study was that the experiment design was perhaps too good: BSS worked well, but so did conventional methods, resulting in no "headroom" for improvement by BSS. They thus could not show that the BSS algorithm in use, fICA (Hyvärinen and Oja, 1997), delivered results superior to those of conventional processing. In a similar set of experiments, Ziehe et al. (2000); Wübbeler et al. (2000) applied the same BSS algorithm as we use below to MEG.

An additional technique that can be applied in an attempt to check whether BSS-based MEG signal processing can significantly surpass conventional techniques is to perform head-to-head comparisons on difficult datasets, meaning datasets with which conventional techniques have difficulty. In order to make such a comparison it is necessary to evaluate the result of the proposed new methods. This evaluation is conducted by checking consistency with known properties of noise, of physiology, and of anatomy. This is a stringent test, as it is unlikely that incorrectly separated data would pass it.

We describe the blind separation problem; review applications of BSS to MEG data with particular emphasis on work in which the authors have been involved; and finish with an evaluation and prognostication. 


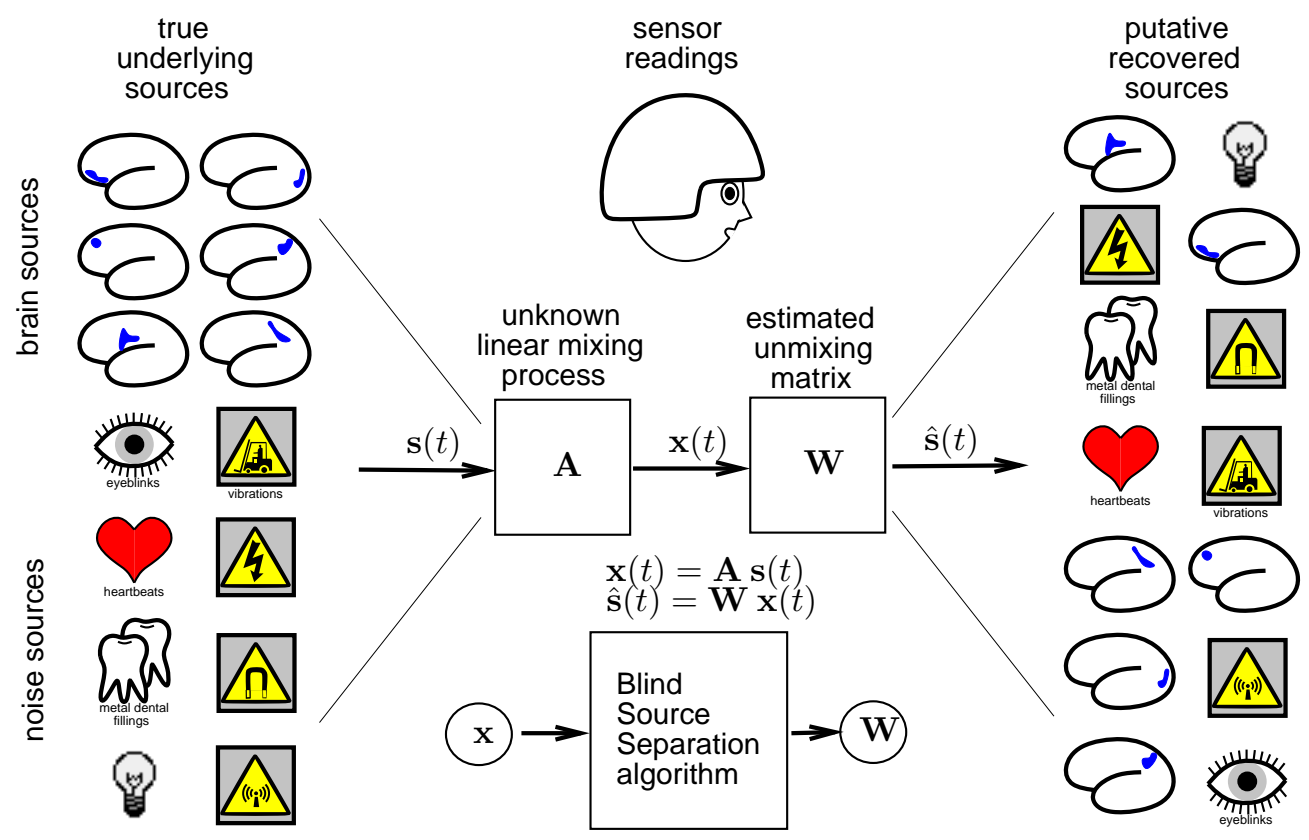

Figure 1: The BSS-for-MEG pipeline. Signals from the brain and other noise sources $\mathbf{s}(t)$ are mixed through an unknown linear mixing process $\mathbf{A}$, resulting in the sensor readings $\mathbf{x}(t)=\mathbf{A s}(t)$. BSS finds an unmixing matrix $\mathbf{W}$ that maps from the sensor signals to recovered components $\hat{\mathbf{s}}(t)=\mathbf{W} \mathbf{x}(t)$. The entries of the attenuation matrix $\mathbf{A}=\mathbf{W}^{-1}$ describe how strongly each sensor responds to each component.

\section{Separation}

Blind separation can be stated as follows. Let $\mathbf{x}(t)$ be an $n$-dimensional vector of sensor signals, which we assume to be an instantaneous linear mixture of $n$ unknown independent underlying sources $s_{i}(t)$, via the unknown stationary $n \times n$ mixing matrix $\mathbf{A}$,

$$
\mathbf{x}(t)=\mathbf{A} \mathbf{s}(t)
$$

The BSS (or ICA) problem is to recover $\mathbf{s}(t)$, given only the measurements $\mathbf{x}(t)$. This is accomplished by finding a matrix $\mathbf{W}$ which approximates $\mathbf{A}^{-1}$, up to permutation and scaling of its rows. After this is accomplished we can find the matrix $\hat{\mathbf{A}}=\mathbf{W}^{-1}$ and denote its columns $\hat{\mathbf{a}}_{i}$. Thus ICA is a decomposition algorithm in the same sense as PCA, in that the data matrix is expressed as a sum of outer products

$$
\left(x_{i}(t)\right)_{i t}=\sum_{j} \hat{\mathbf{a}}_{j}\left(\hat{s}_{j}(t)\right)_{t}^{T}
$$

ICA algorithms come in various classes. Instantaneous algorithms, such as Bell-Sejnowski Infomax (1995) and fICA (Hyvärinen and Oja, 1997), make repeated passes through the dataset and update the unmixing matrix in response to the data at each time point. They are derived under the assumption that the signals are white, and their results should therefore be invariant to shuffling of the data. As a consequence of this, they cannot take advantage of the temporal structure of each source as a cue for correct separation. Summary algorithms first make a pass through the data while summary statistics are accumulated by averaging; they then operate solely upon the summary statistics to find the unmixing matrix W. Summary algorithms should in general be relatively insensitive to sensor noise, because their summary statistics are averages over time. The relatively poor signal-to-noise ratios in MEG data suggested the choice of a summary algorithm. Furthermore noise of interest and neurogenic signals have broad autocorrelation functions, suggesting the use of a non-instantaneous, or contextual (Pearlmutter and Parra, 1996), algorithm. For these reasons we chose a non-instantaneous summary algorithm.

An example of such an algorithm is SOBI (Belouchrani et al., 1993; Cardoso, 1994), which can use the temporal structure of the sources as a cue, and gives high quality separation while imposing rather modest computational requirements. The particular statistics SOBI uses are the correlations between pairs of sensors at a fixed delay, $\mathbf{R}(\tau)=\left\langle\mathbf{x}(t) \mathbf{x}(t+\tau)^{T}\right\rangle$, which are simultaneously approximately diagonalized. SOBI makes good use of abundant but noisy data, and can be tuned by modifying its set of delays, allowing one to gently integrate a very weak form of prior knowledge, namely knowledge of the length 
MEG Image

(a) Subject \#1 component 1 (60Hz contamination)

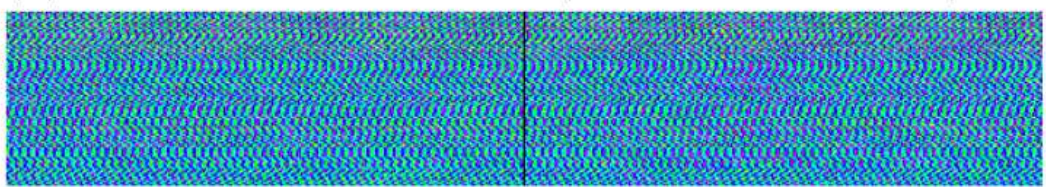

Field Map

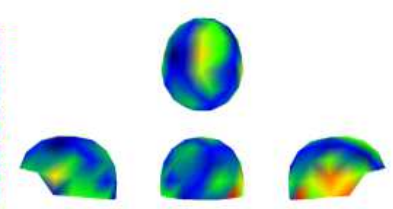

(b) Subject \#1 component 20 (slow DC drift)
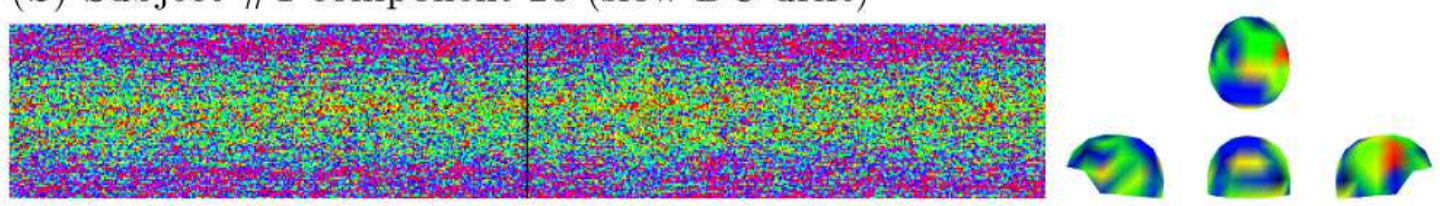

(c) Subject \#1 component 6 (eyeblinks)
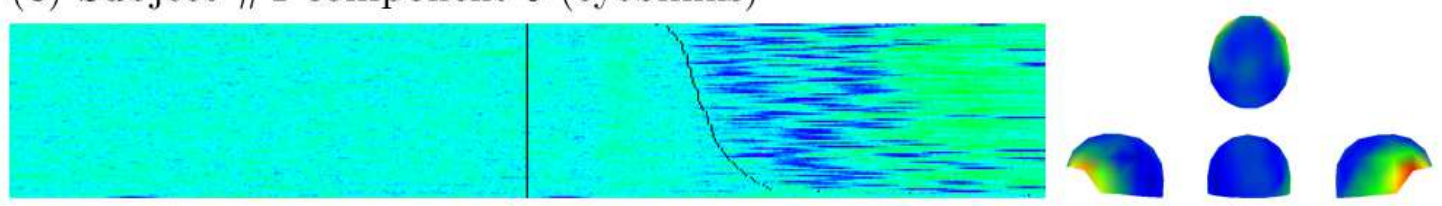

(d) Subject \#1 component 27 (occipital)
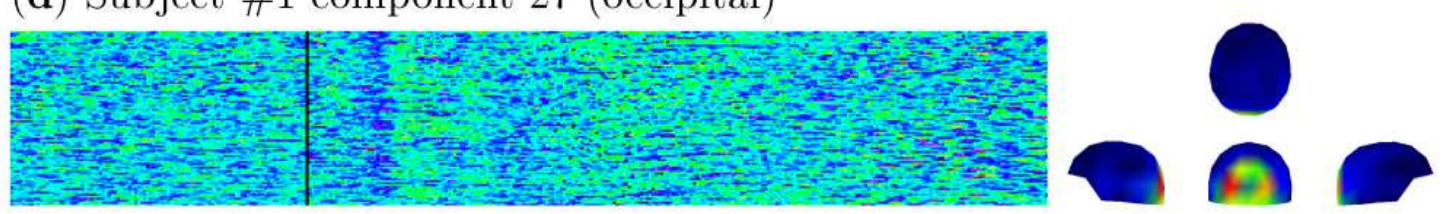

(e) Subject \#1 component 14 (occipital-parietal)
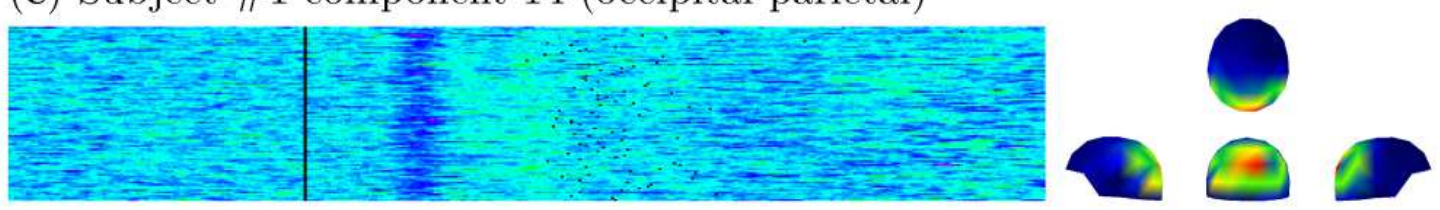

(f) Subject \#1 component 7 (right somatosensory)
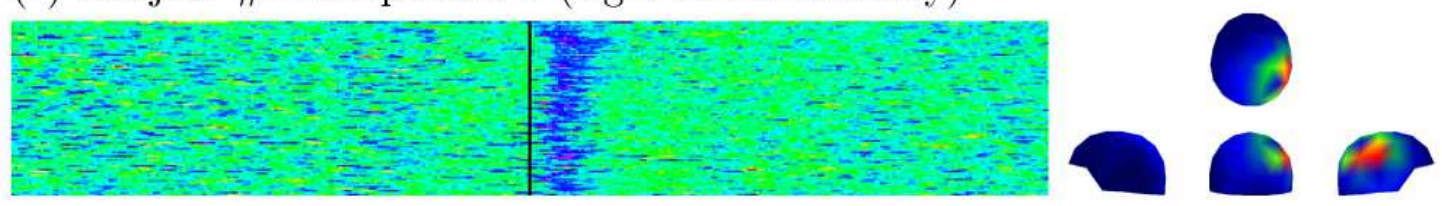

(g) Subject \#1 component 34 (left somatosensory)
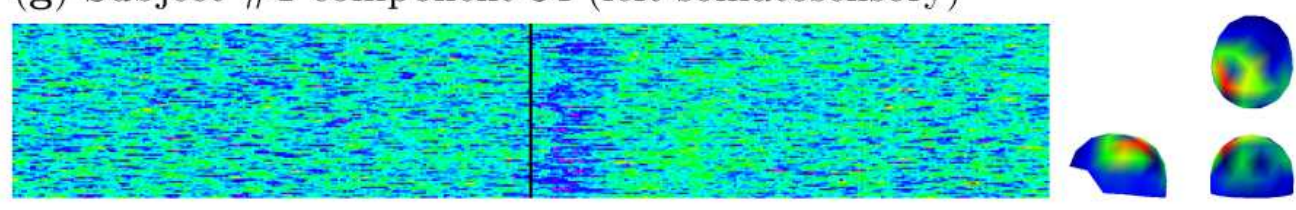

Figure 2: Separation of noise sources and neuronal components using blind source separation. Left: MEG images. Each panel contains 90 trials. Trials are ordered vertically, except for (c), which is sorted by reaction time and where the black curve indicates the times of button presses. Pixels are color-coded source strength. Vertical black lines indicate stimulus onset time. Pre- and post-stimulus durations are each $1000 \mathrm{~ms}$. Right: field maps. Left sagittal, right sagittal, dorsal, and posterior views. False colors indicate the strength with which the component influences the 61 sensor pairs. This figure presents MEG images and field maps of (a) a $60 \mathrm{~Hz}$ component, (b) a slow DC drift component, (c) an eyeblink component, (d) and early occipital visual component, (e) a later occipital-parietal visual component (f) a right somatosensory component and (g) a left somatosensory component. From Tang et al. (2000a). 

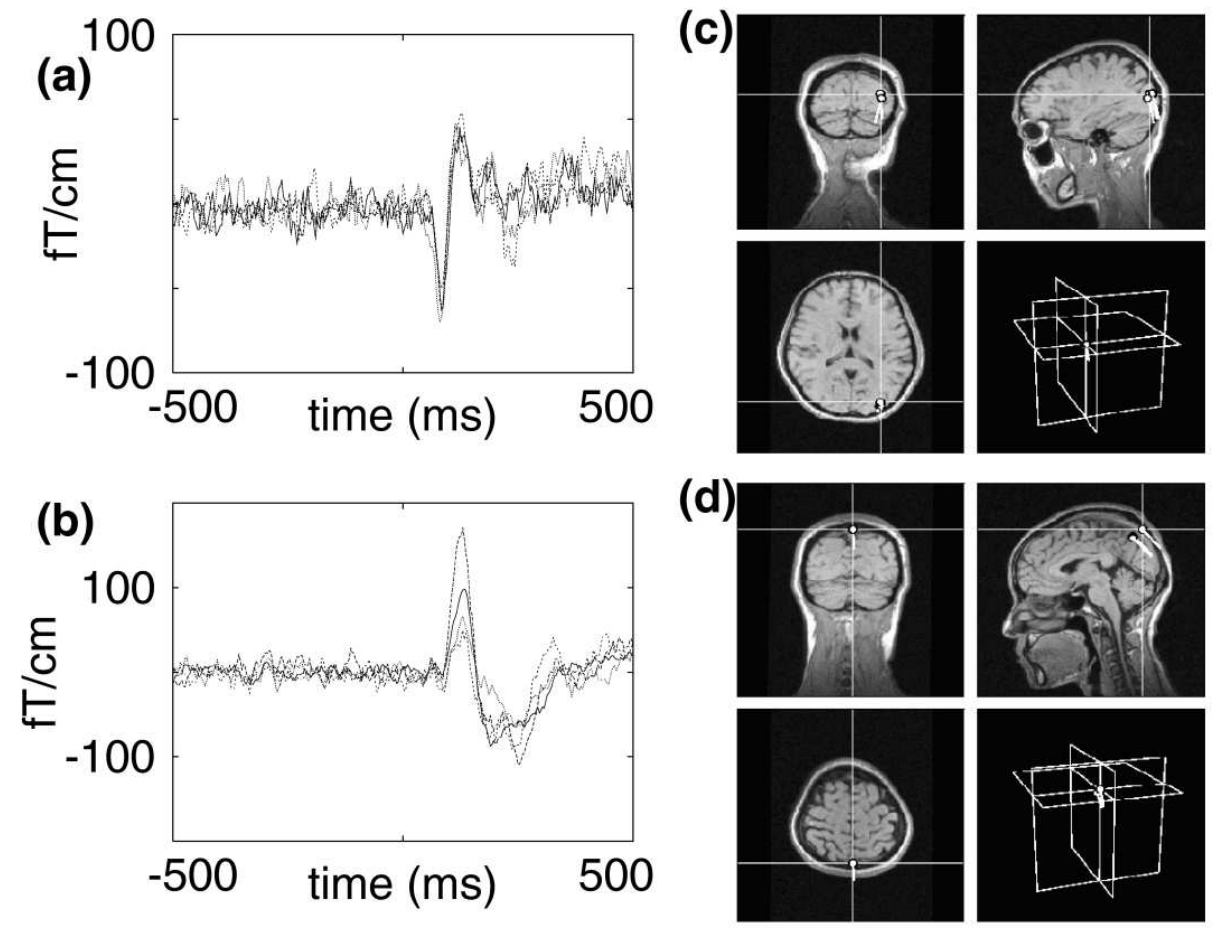

Figure 3: Consistency of separation: overlaid time course and localized dipoles for separated visual components from four similar visual reaction time tasks on a single subject. The primary (fastest) source is shown on the top, and the second-fastest on the bottom. From Tang et al. (2002).

constant of the autocorrelation function.

As shown in Figures 2 and 3, by applying SOBI to MEG data we achieved excellent separation of noise (Tang et al., 2000a), resulting in signal-to-noise improvement sufficient to perform single trial onset detection (Tang et al., 2000b), and we showed that neuronal components can be separated from each other and localized in a robust (across modalities, tasks, and subject) fashion (Tang et al., 2002). These results are surveyed by Tang and Pearlmutter (2002).

\section{Localization}

Separation and localization can be performed jointly or sequentially. Given BSS as a preprocessing step, we would expect a small number of focal regions in each neurogenic component. Conventional methods (Gorodnitsky and Rao, 1997; Mosher and Leahy, 1999) give good performance under such conditions. However they are slow, assume certain noise properties, and generally require manual assistance. Furthermore they are limited in speed by the speed of the forward model, which imposes a surprisingly strong design constraint on MEG machines.

In attempts to build real-time noise-robust localizers,
Jun et al. (2002a,b) take a universal approximator approach to localization. The system is trained to directly map noisy sensor readings to dipole locations. This training results in an MLP localizer which is is not limited (at runtime) by the speed of the forward model. In our case, the training was done by passing random dipoles through a forward model and contaminating the resulting sensor readings with realistic noise. This gives accuracy vaguely matched to that of a conventional algorithm. One can apply a few iterations of conventional Levenberg-Marquardt (LM) minimization using the MLP's output as the initial

\begin{tabular}{ccc}
\hline algorithm & time $(\mathbf{m s})$ & accuracy $(\mathbf{m m})$ \\
\hline 4-start-LM & 448.6 & 11.6 \\
MLP & 0.5 & 11.9 \\
MLP-start-LM & 34.6 & 2.8 \\
\hline
\end{tabular}

Table 1: Real-time performance using an inverse model via universal approximator: a multilayer perceptron is trained to invert the mapping of an analytical forward model. Shown are conventional Levenberg-Marquardt, our MLP, and our hybrid, under real brain noise. Times were measured on a $800 \mathrm{MHz}$ AMD K7. 
guess, resulting in a hybrid MLP-start-LM localizer. As shown in Table 1 , this resulted in a speedup of $900 \times$ with matched accuracies, or a $4 \times$ improved linear accuracy at a speedup of $20 \times$.

These methods works equally well on the LANL SIS Mark I MEG system, which has superconducting magnetic shields/mirrors that complicate the signal path to the point that a forward simulation requires many seconds (Kraus et al., 1998, 2002). Our localizer, in its non-hybrid form, still performs a localization to about the same accuracy in about the same amount of time. The work has also been extended to use a distributed output representation resulting in increased robustness to interfering secondary dipoles and potentially allowing rapid multidipole solutions to be constructed. In addition, the system has been extended to incorporate an extra head-position input allowing for a subject-independent localizer.

\section{Conclusion}

Blind separation of MEG data has been definitively shown to remove various sources of noise. MEG machines are expensive because of heroic technological measures required to raise the SNR. For this reason, it is difficult to justify not performing BSS-based preprocessing on MEG data to improve the SNR, even if separated neuronal sources are to be recombined for conventional processing.

As we have seen, SOBI preprocessing can do more than just remove noise: it can reliably segregate various sources of neuronal activity, allowing more sources to be recovered and localized. The SNR of individual sources is often sufficiently high to allow single-trial analysis, opening the door to a broader range of experiments and, in concert with robust real-time localization methods, potentially allowing the construction of MEG-based braincomputer interfaces.

\section{Acknowledgements}

Supported by US NSF CAREER 97-02-311, the MIND Institute, an equipment grant from Intel corporation, a gift from the NEC Research Institute, and Science Foundation Ireland.

\section{References}

Bell, A. J. and Sejnowski, T. J. (1995). An informationmaximization approach to blind separation and blind deconvolution. Neural Computation, 7(6):1129-1159.

Belouchrani, A., Meraim, K. A., Cardoso, J.-F., and Moulines, E. (1993). Second-order blind separation of correlated sources. In Proc. Int. Conf. on Digital Sig. Proc., pages 346-351, Cyprus.
Cardoso, J.-F. (1994). On the performance of orthogonal source separation algorithms. In European Signal Processing Conference, pages 776-779, Edinburgh.

George, J. S., Aine, C. J., Mosher, J. C., Schmidt, D. M., Ranken, D. M., Schlitt, H. A., Wood, C. C., Lewine, J. D., Sanders, J. A., and Belliveau, J. W. (1995). Mapping function in the human brain with magnetoencephalography, anatomical magnetic-resonanceimaging, and functional magnetic-resonance-imaging. J Clin. Neurophysiol., 12(5):406-431.

Gorodnitsky, I. F. and Rao, B. D. (1997). Sparse signal reconstruction from limited data using FOCUSS: A re-weighted minimum norm algorithm. IEEE Transactions on Signal Processing, 45(3):600-616.

Hämäläinen, M., Hari, R., Ilmoniemi, R. J., Knuutila, J., and Lounasmaa, O. V. (1993). Magnetoencephalography-theory, instrumentation, and applications to noninvasive studies of the working human brain. Rev. Modern Physics, 65:413-497.

Hari, R. (1999). Magnetoencephalography as a tool of clinical neurophysiology. In Niedermeyer, E. and Lopes Da Silva, F., editors, Electroencephalography: Basic Principles, Clinical Applications, and Related Fields. Lippincott Williams \& Wilkins, 4th edition.

Hari, R., Salmelin, R., Makela, J., Salenius, S., and Helle, M. (1997). Magnetoencephalographic cortical rhythms. Int J Psychophysiol, 26(1-3):51-62.

Hyvärinen, A. and Oja, E. (1997). A fast fixed-point algorithm for independent component analysis. Neural Computation, 9(7).

Jun, S. C., Pearlmutter, B. A., and Nolte, G. (2002a). Fast accurate MEG source localization using a multilayer perceptron trained with real brain noise. Physics in Medicine and Biology, 47(14):2547-2560.

Jun, S. C., Pearlmutter, B. A., and Nolte, G. (2002b). MEG source localization using a MLP with a distributed output representation. IEEE Transactions on Biomedical Engineering. To appear.

Jung, T.-P., Humphries, C., Lee, T.-W., McKeown, M. J., Iragui, V., Makeig, S., and Sejnowski, T. J. (2000). Removing electroencephalographic artifacts by blind source separation. Psychophysiology, 37:163-178.

Jung, T.-P., Makeig, S., Westerfield, M., Townsend, J., Courchesne, E., and Sejnowski, T. J. (1999). Analyzing and visualizing single-trial event-related potentials. In Advances in Neural Information Processing Systems 11, pages 118-124. MIT Press.

Kraus, Jr., R. H., Flynn, R. H., Overton, W., Espy, M. A., George, J. S., Matlashov, A., Peters, M. A., and Ru- 
miner, P. (1998). Source localization for a superconducting imaging surface whole-head MEG array. In Yoshimoto, T., editor, Recent Advances in Biomagnetism, 11th Intl. Conf. Biomagnetism, Senai, Japan.

Kraus, Jr., R. H., Volegov, P. L., Maharajh, K., Espy, M. A., Matlashov, A. N., and Flynn, E. R. (2002). Performance of a novel SQUID-based superconducting imaging-surface magnetoencephalography system. Physica C, 368(1-4):18-23.

Lewine, J. D. and Orrison, II, W. W. (1995). Magnetoencephalography and magnetic source imaging. In Orrison, II, W. W., Lewine, J. D., Sanders, J. A., and Hartshorne, M. F., editors, Functional Brain Imaging, pages 369-417. Mosby, St. Louis.

McKeown, M. J., Makeig, S., Brown, G. G., Jung, T.P., Kindermann, S. S., Bell, A. J., and Sejnowski, T. J. (1998a). Analysis of fMRI data by blind separation into independent spatial components. Human Brain Mapping, 6:160-188.

McKeown, M. J., Makeig, S., Brown, G. G., Jung, T. P., Kindermann, S. S., Bell, A. J., and Sejnowski, T. J. (1998b). Analysis of fMRI data by blind separation into independent spatial components. Human Brain Mapping, 6(3):160-188.

Mosher, J. C. and Leahy, R. M. (1999). Source localization using recursively applied projected (RAP) MUSIC. IEEE Transactions on Signal Processing, 47(2):332340.

Pearlmutter, B. A. and Parra, L. C. (1996). A contextsensitive generalization of ICA. In International Conference on Neural Information Processing, pages 151157, Hong Kong. Springer-Verlag.

Tang, A. C. and Pearlmutter, B. A. (2002). Independent components of magnetoencephalography: Localization and single-trial response onset detection. In Kaufmann, L. and Liu, Z. L., editors, Proceedings of Neuromagnetism at the Millennium: A conference to honor Professor Samuel J. Williamson on the occasion of his 60th birthday Friday September 24, 1999, New York University. In press.

Tang, A. C., Pearlmutter, B. A., Malaszenko, N. A., Phung, D. B., and Reeb, B. C. (2002). Independent components of magnetoencephalography: Localization. Neural Computation, 14(8):1827-1858.

Tang, A. C., Pearlmutter, B. A., Zibulevsky, M., and Carter, S. A. (2000a). Blind separation of multichannel neuromagnetic responses. Neurocomputing, 3233:1115-1120.

Tang, A. C., Pearlmutter, B. A., Zibulevsky, M., Hely, T. A., and Weisend, M. P. (2000b). An MEG study of response latency and variability in the human visual system during a visual-motor integration task. In $A d$ vances in Neural Information Processing Systems 12, pages 185-191. MIT Press.

Vigário, R., Jousmäki, V., Hämäläinen, M., Hari, R., and Oja, E. (1998). Independent component analysis for identification of artifacts in magnetoencephalographic recordings. In Advances in Neural Information Processing Systems 10, pages 229-235. MIT Press.

Vigário, R., Särelä, J., Jousmäki, V., Hämäläinen, M., and Oja, E. (2000). Independent component approach to the analysis of EEG and MEG recordings. IEEE Transactions on Biomedical Engineering, 47(5):589-593.

Wübbeler, G., Ziehe, A., Mackert, B.-M., Müller, K.R., Trahms, L., and Curio, G. (2000). Independent component analysis of non-invasively recorded cortical magnetic DC-fields in humans. IEEE Transactions on Biomedical Engineering, 47(5):594-599.

Ziehe, A., Müller, K.-R., Nolte, G., Mackert, B.-M., and Curio, G. (2000). Artifact reduction in magnetoneurography based on time-delayed second order correlations. IEEE Transactions on Biomedical Engineering, 47(1):75-87. 